\title{
Pediatric pain management: the multidisciplinary approach
}

This article was published in the following Dove Press journal:

Journal of Pain Research

8 November 2013

Number of times this article has been viewed

\author{
Shannon Odell ${ }^{1,2}$ \\ Deirdre E Logan ${ }^{1,2}$ \\ 'Division of Pain Medicine, \\ Department of Anesthesiology, \\ Perioperative and Pain Medicine, \\ Boston Children's Hospital, \\ ${ }^{2}$ Department of Psychiatry, Harvard \\ Medical School, Boston, MA, USA
}

Correspondence: Shannon Odell Division of Pain Medicine, Department of Anesthesiology, Perioperative and Pain Medicine, Boston Children's Hospital, 333 Longwood Avenue. Boston, MA 02115 , USA

Tel $+178 \mid 2161668$

Fax $+|78| 216 \mid 652$

Email shannon.odell@childrens.harvard.edu

\begin{abstract}
Chronic pain in children and adolescents is a growing problem and one that is increasingly being addressed with multidisciplinary treatment teams. This review summarizes different multidisciplinary clinics, focusing specifically on intensive pediatric pain rehabilitation centers. This review offers a summary of the challenges faced by these programs and areas for future study.
\end{abstract}

Keywords: pediatric pain rehabilitation, pediatric chronic pain, interdisciplinary, pain associated disability

\section{Introduction}

Chronic pain is a growing problem among children and adolescents, with some epidemiological studies indicating that approximately $30 \%$ of children and adolescents experience pain that lasts for 3 months or longer. ${ }^{1,2}$ The most common pediatric chronic pain complaints include migraine, recurrent abdominal pain, and general musculoskeletal pain, including limb pain and back pain..$^{2-4}$ Chronic pain is often associated with functional disability. In the pediatric population this disability most frequently manifests as school impairment, difficulty maintaining social contacts, decreased participation in recreational activities, impairments in health related quality of life, and an increase in health care utilization. ${ }^{4-6}$ As a result, these patients exact high costs on the health care system. Clinch and Eccleston described childhood chronic pain as "a modern public health disaster."

This complex health problem requires comprehensive treatment approaches. In recent years, multidisciplinary pediatric pain rehabilitation centers have gained increasing support as the treatment of choice for chronic pain conditions. Multidisciplinary teams typically consist of multiple providers of varying specialties who work together to assess and develop a treatment plan for a patient and his or her family. Typical disciplines include medicine, psychology, physical therapy, occupational therapy, nursing, and psychiatry, sometimes in conjunction with alternative medicine practitioners (eg, acupuncture, massage therapy, etc). This treatment approach should be differentiated from the interdisciplinary model, which is also comprised of several different specialists but who work together under a more fluid structure with flexible collaboration and shared treatment goals, which in the case of chronic pain programs, typically emphasize functional restoration. From this point forward, the term "multidisciplinary" will be used to encompass both treatment approaches. 
The intensive multidisciplinary pain rehabilitation approach is based on the biopsychosocial model of pain, which accounts for the complex interplay of the biological, psychological, individual, social, and environmental factors that contribute to and maintain chronic pain symptoms and subsequent disability. Initially created for adult pain patients, these programs have been found to be as cost effective, if not more so, than other medical treatments, and have the added benefit of better outcomes for medication use, health care utilization, functional activities, return to work, and closure of disability claims. ${ }^{8,9}$

This treatment model has begun to gain international favor in the world of pediatrics. In fact, several countries in Europe and certain areas of the United States have begun to mandate that chronic pain be treated with a multidisciplinary approach. However, relatively few of these treatment centers exist worldwide when compared to increasing identification of children suffering from chronic pain. Multidisciplinary centers have large areas of catchment, and families sometimes have to drive long distances to have their child evaluated or treated in one of these centers. ${ }^{10}$

This article pursues three main objectives aimed at familiarizing the reader with the multidisciplinary approach to complex pediatric chronic pain conditions. First, we review the experience of chronic pain and associated disability in children and adolescents. Second, we describe existing models of multidisciplinary pediatric pain programs and their effectiveness, focusing specifically on intensive multidisciplinary pediatric pain programs. Finally, we make recommendations for further areas of clinical development and study.

\section{Chronic pain and disability in children and adolescents}

Vetter published a clinical profile of patients referred to a chronic pain program in Indianapolis. ${ }^{11} \mathrm{He}$ found that enrolled patients were primarily female with chronic pain persisting for 1 year or more, and accompanied by clinically significant anxiety or depression. He also found a significantly disproportionate percentage were from a nonintact biological family (when compared to state and national norms), and 95\% had previously been under the care of at least one other subspecialist for their pain. Most studies have not looked at demographic profiles in such depth; however, other published reports have also noted the predominant number of females enrolled in these programs. The psychological correlates of chronic pain have been well documented, with several studies showing that children and adolescents with persistent pain express higher levels of anxiety and depression, particularly among girls., ${ }^{1,12-15}$ Depressive symptoms in pediatric pain have been linked to more school impairment and perception of pain intensity. ${ }^{16}$ It has also been documented that adolescents with chronic pain report social deficits compared to their peers. ${ }^{17}$

The term "pain associated disability" has been used to describe a chronic pain condition with significant functional impairment, regardless of location or cause. ${ }^{18}$ Discrepancies often exist between the actual tissue damage sustained and the perceived severity of the condition or degree of disability. Factors such as poor coping skills and psychosocial and environmental stressors may contribute to behavioral patterns of pain avoidance, ultimately leading to significant functional disability over time. ${ }^{19}$ Due to the complex nature of the symptoms, families often seek care from multiple providers over time. Therefore, a biopsychosocial model of treatment found in most multidisciplinary pediatric pain centers has been identified as the treatment of choice for such symptoms and disability because this model encompasses the multidimensional features of the chronic pain experience as it affects both the child and the family.

\section{Multidisciplinary program types}

In 1994, the International Association for the Study of Pain published a classification of pain treatment facilities which included multidisciplinary teams. These include modalityoriented clinics, pain clinics specializing in a specific diagnosis or body location, multidisciplinary pain clinics, and multidisciplinary pain centers. The last two include health care professionals who specialize in the assessment and management of patients with a range of painful diagnoses. Pain centers specifically denote research, professional education, and patient care as important components. Members of a multidisciplinary pain team can include physicians (typically anesthesiologists or physiatrists), nurses, psychologists, physical therapists, occupational therapists, recreational therapists, pharmacists, nutritionists, and social workers, among others. The International Association for the Study of Pain curriculum guidelines recommend that the multidisciplinary approach should include appropriate assessment measures for primary domains (including sensory, affective, cognitive, physiological, and behavioral), assess individual factors, sociocultural characteristics of pain, clinical characteristics of pain, impact on quality of life, and impact on daily living. ${ }^{20}$

Parents and their children report higher satisfaction after treatment in a multidisciplinary pain treatment center 
when compared to other previously sought treatments. ${ }^{21}$ Schurman and Friesen found that families perceived that multidisciplinary team members demonstrated interest in understanding the child's pain and spent more time providing information and education about chronic pain conditions. ${ }^{22}$ These findings are important as patient and parent satisfaction are highly correlated with improved adherence to treatment recommendations.

\section{Intensive multidisciplinary pediatric pain rehabilitation programs}

The primary goals of multimodal pain programs in general, and intensive pain rehabilitation centers in particular, are to improve physical functioning and facilitate re-engagement in age typical activities (eg, improving school attendance and social involvement). These intensive rehabilitation programs are being increasingly identified as the treatment of choice for children with chronic pain and associated functional disability and their families. In their worldwide call for an increase in multidisciplinary pain rehabilitation centers, Hechler et al identified the rising rate of chronic pain diagnoses among children and adolescents and the increased health care utilization of these patients as the primary reasoning behind the need for these multimodal programs. ${ }^{23}$ Such programs typically target patients who have failed outpatient therapies and continue to seek medical intervention for their chronic pain condition. Common criteria for admission into these programs include the existence of pain related functional disability. There often must be a parent or caregiver willing to participate in treatment. ${ }^{13,19}$

In a study by Simons et al, researchers examined 100 matched participants, 50 of whom were enrolled in an intensive day hospital pain rehabilitation program and 50 of whom pursued standard outpatient treatments following initial multidisciplinary evaluation of their pain problem. ${ }^{24}$ Intensive intervention included daily physical, occupational, and psychological therapies in addition to medical monitoring for an average of 3-4 weeks, whereas outpatient intervention was comprised of various combinations of medical treatment, physical therapy, and psychological therapies. While there were improvements across both treatment approaches at discharge, patients enrolled in the intensive pain rehabilitation program had significantly larger improvements in all areas measured, including functional disability, pain-related fear, and willingness to adopt a self-management approach to treating pain.

Exercise therapy with treatment by physical therapy (PT) and/or occupational therapy (OT) is a vital component of each of these programs. A PT/OT approach is used for various musculoskeletal conditions and has been demonstrated to be an integral part of the treatment of complex regional pain syndrome. ${ }^{25} \mathrm{PT}$ and OT exercises are typically tailored to the individual patient but can include transcutaneous electrical nerve stimulation, aerobic exercise, stretching, progressive weight bearing, tactile desensitization, massage, and contrast baths. The amount of exercise therapy varies across programs, with Sherry et al finding that an intensive exercise program for patients with complex regional pain syndrome receiving 4-6 hours of aerobic, functionally directed exercise and desensitization showed significant improvements. ${ }^{26}$ After 2 years, $88 \%$ of patients reported being symptom free. However, programs with fewer hours dedicated to exercise have also demonstrated significant improvements in the functional abilities of their patients. ${ }^{12,13,19,27}$

In conjunction with physical therapy, psychological therapy is an integral component of the multidisciplinary pain treatment model. Cognitive-behavioral interventions for pain coping and anxiety and behavioral shaping for the expression of pain-related actions are the primary emphasis in treatment for a majority of multidisciplinary pediatric pain programs. The primary goal of cognitive-behavioral therapy (CBT) treatment for pediatric chronic pain is to teach the child self-regulation techniques through the use of strategies such as relaxation (eg, progressive muscle relaxation, biofeedback, and guided imagery) and cognitive strategies (eg, cognitive restructuring, problem-solving, the creation of fear hierarchies, etc) to help these children perceive their pain and its impact as less debilitating. Techniques also include modifying pain behaviors through operant and behavioral principles. $^{28}$

Eccleston et al, in their examination of a cognitivebehaviorally based residential program, found that immediate treatment effects after a 3 -week program included improved functioning (both objectively and subjectively). ${ }^{27}$ After a 3-month follow-up, improvements included a significant decrease in reports of anxiety, catastrophic thinking about pain, disability, and somatic awareness. School attendance also improved at 3 months, with 27 of the 42 patients included in the analyses improving in at least one category of schooling. These results are consistent with other outcome studies of intensive multidisciplinary pain rehabilitation centers. ${ }^{13,19,29}$ Across programs, delivery models of psychological treatment included individual therapy, group therapy, family therapy, and parent training.

CBT and behavioral modification are the primary theoretical approaches used by these programs; however, 
Acceptance and Commitment Therapy (ACT) is beginning to emerge as another treatment of choice for patients with chronic pain. ACT focuses on engaging in activities regardless of pain, rather than attempting to alleviate the uncomfortable sensations. In adult studies, patients who received ACT reported better emotional, social, and physical functioning, less medication use, and better work status. ${ }^{30}$ Gauntlett-Gilbert et al examined ACT treatment of adolescents with chronic pain in an intensive rehabilitation setting. ${ }^{31}$ They found that an acceptance, values-based treatment approach resulted in improvements in self-reports of functioning. At a 3-month follow-up, these same patients increased school attendance, engaged in less pain catastrophizing, and decreased their health care utilization. Simons et al also explored pain acceptance in both children and parents, finding that greater acceptance was negatively associated with protective parent responses to pain, pain catastrophizing, and parent fear of pain. ${ }^{32}$

Addressing parental responses to the child's pain is another important component of many of these programs, with a growing evidence base supporting the importance of incorporating parents into the treatment. Most intensive rehabilitation programs recognize and address the role of parental factors in the maintenance and exacerbation of their child's chronic pain condition. Recent studies show that parental responses to their child's pain behaviors (eg, protectiveness, minimization of pain, encouraging and monitoring responses) and parent pain catastrophizing are associated with increased functional disability in the child, school impairment, activity restriction, and willingness to engage in treatment. ${ }^{33-38}$ Early studies show that parents whose children are enrolled in these intensive programs improved in their perception of their child's functional disability, as well as improved their own anxiety, depression, and parental stress scores. ${ }^{27}$ Furthermore, parents of children enrolled in these programs were more willing to adopt a self-management approach to pain and reported reductions in their perceptions of their child's fear of pain with treatment. ${ }^{39}$

Although each program has the primary goal of functional improvement, disparities exist in the format, organization, and balance of treatment modalities. A primary distinction is that of inpatient versus day hospital treatment models. Outcomes for each appear similar in the literature to date. In comparing Maynard et al and Hechler et al's outcomes from their inpatient treatment programs to day hospital outcomes, each showed significant changes in pain coping and improvements in functional disability. ${ }^{13,19,40}$ The Cleveland Clinic Children's Hospital's pain rehabilitation program combines an inpatient and day hospital admission for some of their patients based on individual needs; however, no outcomes from this center have been published. Other differences include length of stay and admission of patients by cohort versus a rolling admissions process. For instance the Mayo Clinic and Bath's programs have fixed, 3-week admissions with a group treatment emphasis. In contrast, programs such as those at Boston Children's Hospital and Kennedy Krieger Institute have more flexible length of stay based on the individual needs and progress of the patient. The amount of each therapy a patient receives also varies across programs and across patients within some programs.

Despite the differences that exist among programs, the overall functional improvements documented in published outcomes from these treatment programs are robust and warrant continued examination.

\section{Areas of further exploration}

At present, when compared to the plethora of research that exists for adult pain centers, there is a dearth of research on multidisciplinary pediatric pain rehabilitation centers, and as of the writing of this review, there are particularly few studies looking at the processes or mechanisms that account for the successful outcomes of these programs. Areas for future study should include collaborative research between the multiple disciplines involved in the treatment of chronic pain, as no research of its kind exists in the community of these intensive pediatric pain programs. The absence of multidisciplinary findings speaks to a larger issue, which is the need to examine the mechanisms within these programs that account for the significant functional improvements these patients experience. The research shows that patients in intensive multidisciplinary programs experience rapid and significant physiological, psychological, and functional changes; however, there is an absence of research for why this is the case.

At present, intensive multidisciplinary pediatric pain programs remain relatively disconnected, in that collaboration in research between centers is rare. Future research should focus on clinical trials involving multiple centers utilizing similar protocols. ${ }^{19}$ Working in partnership to coordinate controlled trials of these treatment centers should be a priority in the coming years.

Perhaps one of the areas to examine in an interprogram collaboration would be the varying levels of multidisciplinary treatment (outpatient, partial hospitalization, inpatient hospitalization) and their efficacy. Examining patient profiles, response to treatment, and other mitigating/moderating 
factors may help better determine the varying necessity in levels of care and provide more individualized treatment for a patient and his or her family. Wager et al identified alarming disparities in regard to parental occupation, socioeconomic status, and education when examining patient travel distances to German pediatric pain clinics. ${ }^{10}$ Determining the most efficient and cost-effective way to deliver validated treatment to children with chronic pain and their families could help to create a framework for hospitals around the world to create greater access to multidisciplinary pain centers.

In order to address concerns about catchment areas, Palermo et al have begun a trial of Internet-based interventions for children with chronic pain and their families. This is especially promising as the Internet becomes a growing medium for the delivery of interventions. ${ }^{41}$ Content is primarily focused on CBT-based interventions and emphasizes pain management strategies in the context of participating in more age-typical activities. Short-term outcomes are promising, showing reductions in activity limitations and pain intensity at a 3-month follow-up. However, there were no differences in parental protectiveness or child-reported depression, suggesting that these interventions may have limitations in addressing the more significant psychosocial aspects of managing chronic pain. Obviously, it is far more challenging to find ways to deliver physically-focused components of pain rehabilitation in the electronic format.

One of the primary barriers to admission into multidisciplinary pediatric pain rehabilitation programs is insurance approval and/or reimbursement, particularly in the United States. Palermo argued for the cost effectiveness of these programs, reasoning that a 3-week admission is more cost efficient when compared to years of health care utilization that can result from suffering from chronic pain. ${ }^{42}$ Long-term outcomes may provide further evidence that these programs are able to mitigate the overuse of health care resources by children and families with chronic pain. However, longterm outcomes (more than 1 year) are largely absent in the research as of the writing of this review. Future studies should examine the outcomes of patients who have participated in a multidisciplinary pain program after several years. Hirschfeld et al recently published the 1-year outcomes from their German pediatric pain center, the results of which show that their 3-week, inpatient pediatric pain rehabilitation program improved patients' pain-related disability and school functioning among $56 \%$ of the 167 patients surveyed. ${ }^{29}$ To better add to the literature that exists on these intensive programs, Hechler et al highlighted several areas of future direction. ${ }^{23}$ They argue that larger sample sizes are needed, and longer-term follow-up outcome data is required to determine the effectiveness of these programs.

The growing rate of pain in children and adolescents has created a conundrum for the worldwide medical community, namely how to treat these patients in a way that reduces costs and decreases health care utilization. Based on the literature, multidisciplinary treatment centers and the treatment they provide offer the best hope for these patients. The data is promising for the effectiveness and utility of intensive multidisciplinary pediatric pain rehabilitation centers as a treatment option for those patients who have failed outpatient therapies. Much work is yet to be done in examining the process and best practices among these centers; however, the data that do exist demonstrate that programs such as these are necessary and have helped countless children and their families regain their functioning and resume a healthy developmental trajectory.

\section{Disclosure}

The authors report no conflicts of interest in this work.

\section{References}

1. Fuss S, Pagé G, Katz J. Persistent pain in a community-based sample of children and adolescents. Pain Res Manag. 2011;16(5):303-309.

2. King S, Chambers CT, Huguet A, et al. The epidemiology of chronic pain in children and adolescents revisited: a systematic review. Pain. 2011;152(12):2729-2738.

3. Huguet A, Miró J. The severity of chronic pediatric pain: an epidemiological study. J Pain. 2008;9(3):226-236.

4. Roth-Isigkeit A, Thyen U, Stöven H, Schwartzenberger J, Schmucker P. Pain among children and adolescents: restrictions in daily living and triggering factors. Pediatrics. 2005;115(2):152-162.

5. Petersen S, Hägglöf BL, Bergström EI. Impaired health-related quality of life in children with recurrent pain. Pediatrics. 2009;124(4); e759-e767.

6. Eccleston C, Bruce E, Carter B. Chronic pain in children and adolescents. Paediatr Nurs. 2006;18(10):30-33.

7. Clinch J, Eccleston C. Chronic musculoskeletal pain in children: assessment and management. Rheumatology (Oxford). 2009;48(5): 466-474.

8. Stanos S. Focused review of interdisciplinary pain rehabilitation programs for chronic pain management. Curr Pain Headache Rep. 2012;16(2):147-152.

9. Turk DC. Clinical effectiveness and cost-effectiveness of treatments for patients with chronic pain. Clin J Pain. 2002;18(6):355-365.

10. Wager J, Ruhe A, Hirschfeld G, et al. Influence of parental occupation on access to specialized treatment for pediatric chronic pain: a retrospective study. Schmerz. 2013;27(3):305-311.

11. Vetter TR. A clinical profile of a cohort of patients referred to an anesthesiology-based pediatric chronic pain medicine program. Anesth Analg. 2008;106(3):786-794.

12. Hechler T, Dobe M, Kosfelder J, et al. Effectiveness of a 3-week multimodal inpatient pain treatment for adolescents suffering from chronic pain: statistical and clinical significance. Clin J Pain. 2009;25(2): $156-166$.

13. Logan DE, Carpino EA, Chiang G, et al. A day-hospital approach to treatment of pediatric complex regional pain syndrome: initial functional outcomes. Clin J Pain. 2012;28(9):766-774. 
14. Hyams JS, Burke G, Davis PM, Rzepski B, Andrulonis PA. Abdominal pain and irritable bowel syndrome in adolescents: a community-based sample. J Pediatr. 1996;129(2):220-226.

15. Simons LE, Sieberg CB, Claar RL. Anxiety and impairment in a large sample of children and adolescents with chronic pain. Pain Res Manag. 2012;17(2):93-97.

16. Logan DE, Simons LE, Kaczynksi KJ. School functioning in adolescents with chronic pain: the role of depressive symptoms in school impairment. J Pediatr Psychol. 2009;34(8):882-892.

17. Eccleston C, Wastell S, Crombez G, Jordan A. Adolescent social development and chronic pain. Eur J Pain. 2008;12(6):765-774.

18. Bursch B, Walco GA, Zeltzer L. Clinical assessment and management of chronic pain and pain-associated disability syndrome. J Dev Behav Pediatr. 1998;19(1):45-53.

19. Maynard CS, Amari A, Wieczorek B, Christensen JR, Slifer KJ. Interdisciplinary behavioral rehabilitation of pediatric pain-associated disability: retrospective review of an inpatient treatment protocol. J Pediatr Psychol. 2010;35(2):128-137.

20. Recommendations for Pain Treatment Services. IASP-Pain.org [homepage on the Internet]. Washington DC: International Association for the Study of Pain. Available from: http://www.iasp-pain.org/AM/Template. cfm?Section=Pain_Treatment_Facilities. Accessed April 15, 2013.

21. Gorodzinsky AY, Tran ST, Medrano GR, et al. Parents' initial perceptions of multidisciplinary care for pediatric chronic pain. Pain Res Treat. Epub August 21, 2012.

22. Schurman JV, Friesen CA. Integrative treatment approaches: family satisfaction with a multidisciplinary paediatric abdominal pain clinic. Int J Integr Care. 2010;10.

23. Hechler T, Dobe M, Zernikow B. Commentary: A worldwide call for multimodal inpatient treatment for children and adolescents suffering from chronic pain and pain-related disability. J Pediatr Psychol. 2010;35(2):138-140.

24. Simons LE, Sieberg CB, Pielech M, Conroy C, Logan DE. What does it take? Comparing intensive rehabilitation to outpatient treatment for children with significant pain related disability. J Pediatr Psychol. 2013;38(2):213-223.

25. Lee BH, Scharff L, Sethna NF, et al. Physical therapy and cognitivebehavioral treatment for complex regional pain syndromes. J Pediatr. 2002;141(1):135-140.

26. Sherry DD, Wallace CA, Kelley C, Kidder M, Sapp L. Short- and longterm outcomes of children with complex regional pain syndrome type I treated with exercise therapy. Clin J Pain. 1999;15(3):218-223.

27. Eccleston C, Malleson PN, Clinch J, Connell H, Sourbut C. Chronic pain in adolescents: evaluation of a programme of interdisciplinary cognitive behavior therapy. Arch Dis Child. 2003;88(10):881-885.

28. Palermo TM. Cognitive-Behavioral Therapy for Children and Adolescents. New York, NY: Oxford University Press; 2012.

29. Hirschfeld G, Hechler T, Dobe M, et al. Maintaining lasting improvements: one-year follow-up of children with severe chronic pain undergoing multimodal inpatient treatment. J Pediatr Psychol. 2013;38(2):224-236.
30. McCracken LM, Eccleston C. A prospective study of acceptance of pain and patient functioning with chronic pain. Pain. 2005;118(1-2): 164-169.

31. Gauntlett-Gilbert J, Connell H, Clinch J, McCracken LM. Acceptance and values-based treatment of adolescents with chronic pain: outcomes and their relationship to acceptance. J Pediatr Psychol. 2013;38(1): 72-81.

32. Simons LE, Sieberg CB, Kaczynski KJ. Measuring parent beliefs about child acceptance of pain: a preliminary validation of the Chronic Pain Acceptance Questionnaire, parent report. Pain. 2011;152(10): 2294-2300.

33. Simons LE, Claar RL, Logan DL. Chronic pain in adolescence: parental responses, adolescent coping, and their impact on adolescent's pain behaviors. J Pediatr Psychol. 2008;33(8):894-904.

34. Goubert L, Vervoort T, Sullivan MJ, Verhoeven K, Crombez G. Parental emotional responses to their child's pain: the role of dispositional empathy and catastrophizing about their child's pain. J Pain. 2008;9(3):272-279.

35. Caes L, Vervoort T, Eccleston C, Vandenhende M, Goubert L. Parental catastrophizing about child's pain and its relationship with activity restriction: the mediating role of parental distress. Pain. 2011;152(1): 212-222.

36. Sieberg CB, Williams S, Simons LE. Do parent protective responses mediate the relation between parent distress and child functional disability among children with chronic pain? J Pediatr Psychol. 2011;36(9): 1043-1051.

37. Guite JW, Logan DE, Simons LE, Blood EA, Kerns RD. Readiness to change in pediatric chronic pain: initial validation of adolescent and parent versions of the Pain Stages of Change Questionnaire. Pain. 2011;152(10):2301-2311.

38. Claar RL, Simons LE, Logan DE. Parental responses to children's pain: the moderating impact of children's emotional distress on symptoms and disability. Pain. 2008;138(1):172-179.

39. Logan DE, Conroy C, Sieberg CB, Simons LE. Changes in willingness to self-manage pain among children and adolescents and their parents enrolled in an intensive interdisciplinary pediatric pain treatment program. Pain. 2012;153(9):1863-1870.

40. Hechler T, Blankenburg M, Dobe M, Kosfelder J, Hübner B, Zernikow B. Effectiveness of a multimodal inpatient treatment for pediatric chronic pain: a comparison between children and adolescents. Eur J Pain. 2010;14(1):97.

41. Palermo TM, Wilson AC, Peters M, Lewandowski A, Somhegyi H. Randomized controlled trial of an Internet-delivered family cognitivebehavioral therapy intervention for children and adolescents with chronic pain. Pain. 2009;146 (1-2):205-213.

42. Palermo TM. Impact of recurrent and chronic pain on child and family daily functioning: a critical review of the literature. J Dev Behav Pediatr. 2000;21(1):58-69.
Journal of Pain Research

\section{Publish your work in this journal}

The Journal of Pain Research is an international, peer-reviewed, open access, online journal that welcomes laboratory and clinical findings in the fields of pain research and the prevention and management of pain. Original research, reviews, symposium reports, hypothesis formation and commentaries are all considered for publication.

\section{Dovepress}

The manuscript management system is completely online and includes a very quick and fair peer-review system, which is all easy to use. Visit http://www.dovepress.com/testimonials.php to read real quotes from published authors. 